\title{
Towards a Scalable IOTA Tangle-Based Distributed Intelligence Approach for the Internet of Things
}

\author{
Tariq Alsboui, Yongrui Qin, Richard Hill, and Hussain Al-Aqrabi \\ School of Computing and Engineering, University of Huddersfield, Huddersfield, UK, \\ tariq.alsboui@hud.ac.uk, y.qin2@hud.ac.uk, r.hill@hud.ac.uk, \\ h.al-aqrabi@hud.ac.uk
}

\begin{abstract}
Distributed Ledger Technology (DLT) brings a set of opportunities for the Internet of Things (IoT), which leads to innovative solutions for existing components at all levels of existing architectures. IOTA Tangle has the potential to overcome current technical challenges identified for the IoT domain, such as data processing, infrastructure scalability, security, and privacy. Scaling is a serious challenge that influences the deployment of IoT applications. We propose a Scalable Distributed Intelligence Tangle-based approach (SDIT), which aims to address the scalability problem in IoT by adapting the IOTA Tangle architecture. It allows the seamless integration of new IoT devices across different applications. In addition, we describe an offloading mechanism to perform proof-of-work (PoW) computation in an energy-efficient way. A set of experiments has been conducted to prove the feasibility of the Tangle in achieving better scalability, while maintaining energy efficiency. The results indicate that our proposed solution provides highly-scalable and energy efficient transaction processing for IoT DLT applications, when compared with an existing DAG-based distributed ledger approach.
\end{abstract}

Key words: Scalability, Distributed Ledger Technology (DLT), IOTA Tangle, Internet of Things (IoT), Distributed Intelligence (DI)

\section{Introduction}

Internet of Things (IoT) applications connect everyday objects to the Internet and enable the gathering and exchange of data to increase the overall efficiency of a common objective [1]. It is estimated that there will be approximately 125 billion devices connected to the Internet in $2030[2,3,4]$. Consequently, most IoT applications are required to be highly scalable and energy efficient, so that they are capable of dynamically responding to a growing number of IoT devices [5].

IoT applications have a number of common elements: (1) sensing to perceive the environment; (2) communication for efficient data transfer between objects, and (3) computation, which is usually performed to generate useful information from the raw data. 
Distributed Intelligence (DI) is an approach that could address the challenges presented by the proliferation of IoT applications. DI is a sub-discipline of artificial intelligence that distributes processing, enabling collaboration between smart objects, and mediating communications, thus supporting IoT system optimisation and the achievement of goals [6]. This definition is the basis for the research that is described in this article.

Contribution: We propose a system architecture for IoT, called the Scalable Distributed Intelligence Tangle-based Approach (SDIT). This research successfully addresses some of the technical challenges presented by the IoT, whilst also supporting the necessary proof-of-work (PoW) mechanism in an energy-efficient way. The key contributions are summarised as follows:

- A Tangle-based architecture that manages resources and enables the deployment of IoT applications with the primary motivation being scalability;

- A task offloading mechanism for performing the proof-of-work (PoW) on powerful IoT, devices to minimise energy consumption when resources (such as power) are constrained;

- A set of experimental results that verify the effectiveness and contribution of the proposed approach.

The ultimate objective of this paper is to design, and develop a scalable and energy efficient IOTA Tangle-enabled IoT intelligent architecture to support DI. The proposed approach differs from other solutions by using a Tangle-based DLT with the primary motivation of being energy-efficient, and scalable to accommodate the growth of IoT while taking resource constrains into consideration.

This work outlines the design of a scalable system that can be used in various IoT applications. IOTA Tangle is used to achieve scalability and a higher level node is responsible for performing the proof of work (PoW) to minimise energy consumption of IoT devices. The initial idea can be found in our previous positioning work [5].

The reminder of this paper is organised as follows: Section 2 describes distributed ledger technology from the perspective of IOTA. Section 3 presents the suitability of IOTA Tangle for IoT. Section 4 discusses the differences of our work from other closely related work. In Section 5 we present our proposed SDIT system architecture. The performance of the proposed implementation is evaluated in Section 6. Finally, we conclude this paper and present an interesting future directions in Section 7.

\section{Distributed Ledger Technology}

Distributed Ledger Technology (DLT) can be divided into three main types based on the differentiation of the data structure used for the ledger, including: BlockChain (BC) [7], IOTA Tangle (DAG) [8], and Hashgraph [9]. BC is a distributed, decentralised, and immutable ledger for storing transactions and sharing data among all network participants [10]. Hashgraph, is considered as 
an alternative to $\mathrm{BC}$ and is used to replicate state machines, which guarantees Byzantine fault tolerance by specifying asynchrony and decentralization, as well as no need for proof-of-work (PoW), eventual consensus with probability of one, and high speed in the consensus process [11]. BC has been criticized for its cost, energy consumption and lack of scalability.

To overcome these limitations, the IOTA Tangle technology has been introduced as a decentralized data storage architecture and a consensus protocol, based on a Directed Acyclic Graph (DAG). Each node in the DAG represents a transaction, and the connections between transactions represent the transaction validators [8].

$\mathrm{BC}$ technology recently started to receive attention from both academic and industry, since it offers a wide range of potential benefits to areas beyond cryptocurrency (in particular the IoT), as it has unique characteristics such as immutability, reliability, fault-tolerance, and decentralization [12]. It is predicted that $\mathrm{BC}$ will transform the IoT ecosystems by enabling them to be smart and more efficient. According to the International Data Cooperation (IDC), it is stated that 20 per cent of IoT deployments will employ a basic level of BC enabled services [13]. This number will continue to increase for the adoption of $\mathrm{BC}$ in the IoT since it is in the early stages of innovation. Overall, $\mathrm{BC}$ is considered as an effective solution to be integrated with the IoT to achieve some of the IoT technical challenges [14].

$\mathrm{BC}$ is potentially able to overcome some of the IoT issues such as privacy and security [15]. However, building an energy-efficient, and scalable IoT applications remains a challenge. Firstly, all BC consensus mechanisms in either private or public BC, require all fully participating nodes to retain copies of all transactions recorded in the history of $\mathrm{BC}$, which comes at the cost of scalability [12]. Furthermore, IoT devices have limited computational, memory and networking constraints, which brings an issue when using BC-based architectures. Some of the IoT devices will not be able to engage in performing Proof of Work (PoW) consensus operations due to their limited computational power and battery life. Also, IoT devices do not always come with the required storage space to place a complete copy of the BC [16].

With the IOTA Tangle, transactions are directly attached to the chain without the need to wait as they need to approve two previous transactions called tips. Hence, the Tangle is more efficient than traditional BC under the welldesigned consensus mechanisms [17].

\section{The IOTA Tangle Suitability for IoT}

The IOTA Tangle is intuitively understandable, and the benefits offered by it can be employed to realise a DI approach. It offers a wide range of prospective modifications to fit specific goals. The scalability and flexibility essential for IoT can be obtained by IOTA technology. IOTA can facilitate IoT interactions in the form of transactions. The following are the potential benefits and motivations for integrating the IOTA technology in the IoT infrastructure: 
- Scalability: IoT demands scalable infrastructure to cope with the increasing number of IoT applications. The IOTA Tangle private network offers high scalability due to the unique design of the decentralized consensus Tanglebased architecture, in which users are also validators, and has no scaling limitations;

- Decentralization: in centralized network architectures, the exchange of data is validated and authorized by central third-party entities. This leads to a higher cost in relation to centralized server maintenance. In IOTA Tangle based architecture, nodes exchange transactions with each other without relying on a central entity. Therefore, any participants who want to exchange transaction on the Tangle need to actively engage in consensus operations;

- Security and privacy: one of the most critical technical challenges of coping with IoT is related to network security and privacy. In order to ensure confidentiality and data protection, IOTA technology has developed Masked Authenticated Messaging (MAM) as a second layer data communication protocol that provides the ability to transmit and access encrypted data streams over the Tangle. MAM encryption is enabled by three modes to control visibility and access to channels including: public, private, and restricted. Consequently, it can encrypt, authenticate, and broadcast data to the IOTA network;

- Zero-fees transactions: IOTA does not require miners as IOTA participants to perform the proof-of-work (PoW) themselves. The transaction cost is regarded as the electricity required to validate two previous transactions in the working mechanism. This means that all network participants are required to contribute their computational power to maintain the network, thus removing transaction fees. The Tangle method allows IOTA to operate fee-free, making the network even more distributed;

- Energy-efficiency: IoT devices have limitations in terms of power consumption, and applications have to be developed to maximise energy efficiency in order to extend device and network life. IOTA technology enables proofof-work (PoW) to be outsourced to a more powerful device to reduce the energy consumption of constrained IoT devices;

- Resiliency: IoT applications require integrity in the data being transmitted and analyzed, therefore IoT infrastructure is required to be resilient against data leaks and breakage (i.e, offline capability). An IOTA network has replicas of records stored over IOTA peers. This assists the maintenance of data integrity, and together with offline tangle capability, provides additional resilience for the IoT infrastructure;

\section{Related Work}

Recently, DI gained new momentum from researchers to overcome the technical challenges of IoT $[18,19,20,21,22,23]$. A distributed dataflow programming model to enable DI is proposed in [18]. The system consists of fog devices that are classified according to their computing resources, edge IO (input/output), 
and compute nodes. The input nodes are capable of brokering communications and data transfer to compute nodes. The compute nodes are responsible for processing the data arriving from input devices. The decisions of assigning logical nodes to physical devices are based on the capability of the nodes and the system designer. The proposed architecture achieves scalability, mobility, and can cope with heterogeneity. However, privacy, offline capability, and resource efficiency are not considered in their design and the approach is not suitable for timecritical applications that require fast responses.

An approach named as PROTeCtPrivacy aRquitecture for integratiOn of internet of Things and Cloud computing to enable DI is presented in [19]. The proposed approach consists of IoT devices and a cloud platform. The IoT devices are responsible for sensing and implementing a cryptographic mechanism i.e, a symmetric algorithm to ensure privacy, before transmitting the data to a cloud. Similarly, in [24], the authors presented an approach based on Mobile Cloud Computing to support DI. The main idea is to merge sensing and processing at different levels of the network by sharing the applications workload between the server side and the smart things, using a cloud computing platform when needed. The proposed approach enables real-time monitoring and analysis of all the acquired data by networked devices and provides flexibility in executing the application by using resources from cloud computing services. However, these approaches are neither scalable nor suitable for time-critical applications. Furthermore, resiliency of the system i.e, offline capability, multi-party authentication for data security [25], and the fusion of data sources from external devices is not considered in their design.

More advanced approaches are proposed in $[20,21,23,26]$ in which they rely on fog computing to enable DI, for example the work presented in [20], in which the authors applied two techniques, device-driven and human driven intelligence to reduce energy consumption and latency. The approach relies on machine learning (ML) to detect user behaviors and adaptively adjusts the sampling rate of sensors and resource schedules (timeslots in the MAC layer) between sensor nodes. Furthermore, an algorithm was designed to deal with the offloading of local tasks among a cluster of fog nodes to further reduce energy consumption, which may reduce energy demands and system latency. However, the approach is not scalable, interactions and information sharing among sensor nodes is not explicitly defined, and it lacks the mechanisms to deal with privacy and offline processing capability.

An architecture that is composed of three layers is proposed in [26]. The approach employs several technologies to achieve DI at different layers. It consists of three layers, each of which is responsible for managing a specific task. The first layer consists of IoT devices and sensor devices, which are responsible for measuring and capturing environmental data. The second layer comprises fog nodes, which is responsible for providing an offloading path for the data captured from a group of IoT devices. The third layer is the cloud, which is responsible for managing computing resources and data, and provides overall control and monitoring of the application. The proposed approach leads to a reduction in 
energy consumption and latency. However, cooperation amongst the physical devices is not provided, and issues related to privacy [27], and offline capability is not considered.

In [21], the authors present a novel three tier architecture to support DI. In the three tier architecture, IoT components such as sensors, mobile phones, vehicles, base station, network connection, and management elements are connected in a multi-tier distributed schema consisting of different levels of intelligence named as follows: group of devices tier, regional tier, and global tier. The group of devices tier consists of IoT devices and is responsible for managing distributed services (data) generated by sensors. The regional tier is made up of fog colony nodes that are considered as intermediate nodes and responsible for data preprocessing and integration. The global tier consists of cloud data centres that are responsible for further data processing. The proposed approach is robust and reduces the cost of maintaining the fog computing paradigm. However, it lacks scalability, resource utilisation mechanisms and privacy, which are considered major challenges in IoT. Furthermore, it uses a predetermined static orchestration, which results in the failure of the system due to depletion of their energy.

Another architecture is proposed in [22] to support DI called Distributed Internet like ArchiTecture (DIAT). The architecture is divided into three layers including: virtualization of physical objects (VO), corresponding virtual object (CVOL), and service level (SL) all of which have their own functionalities and responsibilities. The virtualization of a physical objects layer provides a semantic description of the capabilities and features of the associated real world objects. The second layer is responsible for communicating and coordinating tasks coming from the VO layer. Finally, the service layer (SL) is responsible for creating and management of services and it handles various requests from users. The proposed architecture is scalable, interoperable and privacy is considered. However, support of the other IoT technical challenges i.g, offline capability, and the conservation of IoT resources is not supported.

Another approach is introduced in [23] where the authors have developed several layers to achieve DI. The approach is divided into four layers including: A first layer is cloud computing that consists of a data centre for providing wide monitoring and centralized control. The second layer comprises intermediate computing nodes that are responsible for identifying dangerous events that provide and act upon them. A third layer comprises low power and high performance edge devices connected to a group of sensors that are responsible for handling the raw data coming from sensors and perform analysis in a timely manner. Finally, the fourth layer consists of sensor nodes distributed to monitor the environment. The advantage of this approach is optimal responses in real time, and low latency. However, IoT related issues such as, energy efficiency, scalability, privacy [28] are not considered in their design.

Most recently, a DAG-based scalable transactive smart home infrastructure is proposed in [29]. The approach adopts IOTA tangle to build an IoT smart home infrastructure focusing on scalability and security. A network of 40 nodes were established and divided into three main parts including: smart homes, the 
Tangle of inter-house transactions (TXs), and smart devices in the homes. In all smart home devices, there is an always an online computation device ("Home Node") with pre-installed firmware and a corresponding tangle reference implementation. All home nodes are connected to their neighbour with TCP/UDP protocols for communication and synchronizing the distributed ledger. The approach is scalable to a small number of nodes, and would consume a lot of energy since all nodes are required to perform the proof-of-work. Also, other IoT-related issues such as offline capability is not considered in their design. Furthermore, since their approach relies on a coordinator, full decentralization is not achieved.

The DAG-based smart home approach is similar to the SDIT approach proposed in this paper. Both approaches utilise IOTA Tangle with different number of nodes to achieve scalability, where our approach focuses more on energy efficiency of constrained IoT devices as well as maintaining a decentralized architecture.

\section{SDIT: A Scalable Distributed Intelligence Tangle-Based Approach}

In this section, we present our proposed Scalable Distributed Intelligence Tanglebased approach (SDIT) that aims at tackling the scalability, energy-efficiency, and decentralisation by adopting the IOTA Tangle technology.

\subsection{SDIT: System Architecture}

Figure 1 illustrates an abstract view picture of the proposed system architecture. The architecture is divided into three main parts including: IoT devices, Tangle to process transactions(txs), and PoW enabled server. Each IoT device is connected with neighbour nodes via TCP/IP protocols for communication, and interaction with the Tangle is in the form of transactions. Tangle is responsible for managing, collecting and processing the transactions. A PoW-enabled server has rich resources and is mostly responsible for performing all of the computations on behalf of the IoT devices. This is a critical task so as to minimise energy consumption. The Tangle can act as a data management layer for processing and storing data in an efficient way. However, the management of data processing is beyond the scope of this paper. The green boxes in Figure 1 represent fully confirmed transactions, which means that they are approved by all of the current tips, whereas the red boxes are not confirmed transactions. The blue boxes are the tips.

\subsection{Consensus Mechanism Employed}

Since we are utilising the IOTA Tangle to deal with transactions, we follow the same working principals in which a new transaction should choose two previous unapproved transactions, which are called Tips, to approve based on the tip 


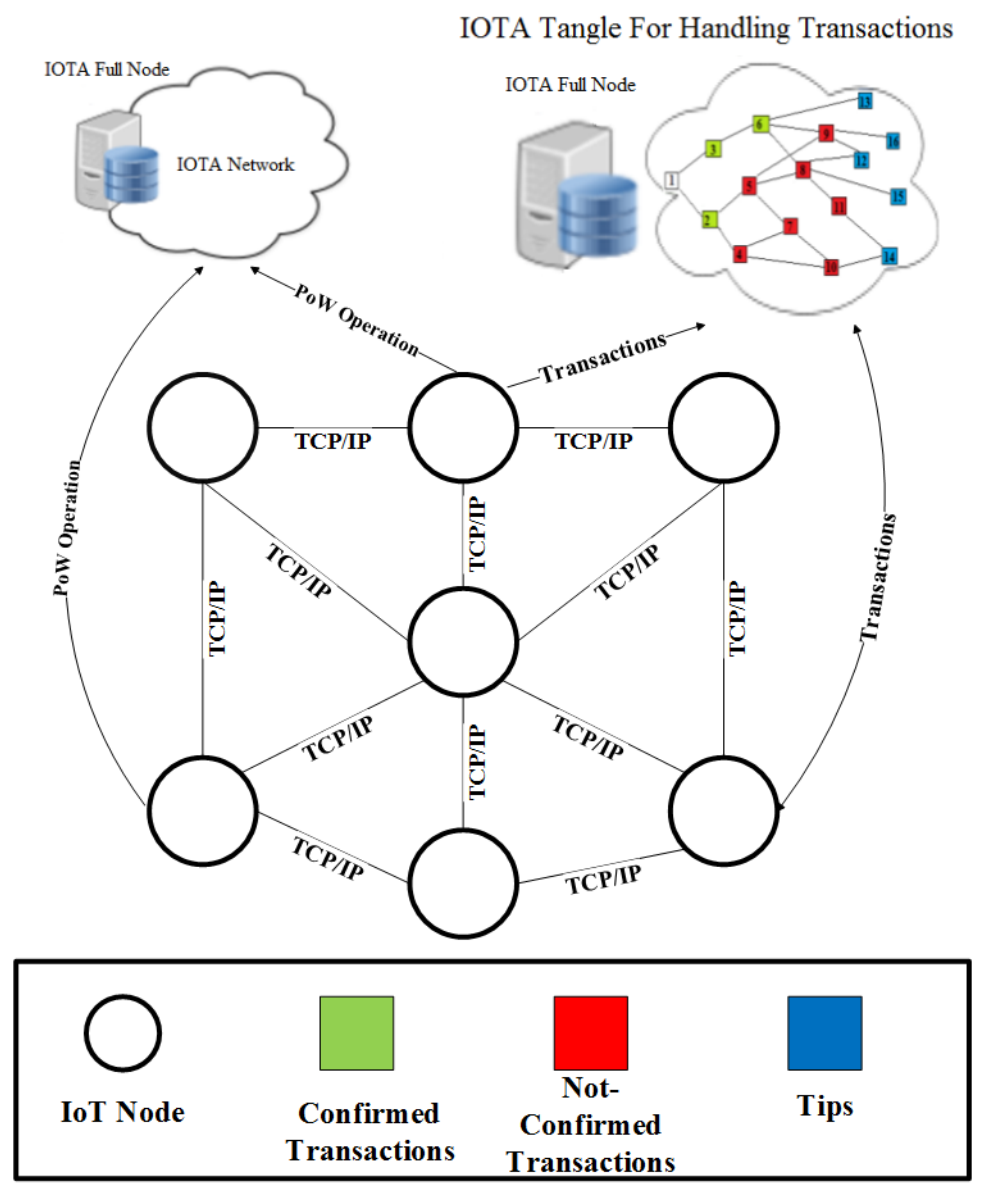

Fig. 1. The Scalable Distributed Intelligence Tangle-based approach (SDIT)

selection algorithm. After tips are selected, the IOTA nodes are able to publish their new transactions to the Tangle. In the advanced Markov Chain Monte Carlo Tip Selection Algorithm (MCMC) $N$ independent random walks are generated on the tangle; the walks begin at the genesis or at a random node and keep moving along the edges of the tangle based on a probability function.

The MCMC Algorithm ensures that the tips are selected non-deterministically along the path of the largest cumulative weight for a reasonable amount of time. The probability from transaction walking from the genesis $L_{x}$ to a tip $L_{y}$ is proportional to $P\left(-\alpha\left(L_{x}-L_{y}\right)\right)$, where $P_{x y}$ is an increasing function (generally an exponential), $\alpha$ is a constant and $c_{i}$ represents the Cumulative Weight of transaction $i$. The process ends when the walker reaches a tip, which is then selected for approval. 
Typically, the first tip is usually selected for approval. For further details on the working mechanism of the MCMC algorithm, we refer the interested reader to the IOTA white paper in [8]. To support the advanced tip selection process, the MCMC technique [8], applies a set of rules for deciding the probability of each step in a random walk, and works as follows:

1. Run the MCMC algorithm $N$ times to choose 100 new transactions (tips). The probability of the transaction being accepted is therefore $M$ of $N$ ( $M$ is the number of times a tip is reached that has a direct path to the transaction);

2. Calculate how many tips that are directly or indirectly connected to a particular transaction and decide with what probability transactions will be accepted as follows:

a) if it is less than $50 \%$, the transaction is not approved as yet (not confirmed);

b) if it is above $50 \%$, the transaction has a fair chance to be approved (awaiting to be confirmed);

c) if it reaches the level of $98 \%$ or $100 \%$, the transaction is considered approved (fully confirmed).

\subsection{Computation Offloading}

Computation offloading can be divided into two categories: data offloading and computation offloading. The former refers to the use of novel network techniques to transmit mobile data originally planned for transport via cellular networks. The latter refers to the offloading of heavy computation tasks to conserve resources [30]. It is commonly assumed that the implementation of computation offloading depends heavily on the design of the network architecture. The main goal of offloading is to reduce total energy consumption or overall task execution time, or both of them.

The IOTA PoWbox (Proof of Work box) is a service provided by the IOTA Foundation that allows the offloading of the PoW to nodes with rich resources, thus reducing the energy consumption of constrained IoT devices and speeding up the development workflow [31]. It was suggested by the authors in [32] to conserve energy of IoT devices by performing the proof-of-work operation offline on a device with rich resources, thus achieving improved energy-efficiency. Figure 2 illustrates the computation offloading mechanism used in the SDIT approach in which the computation operation of performing the $\mathrm{PoW}$ is offloaded to a device with higher resources. This leads to a reduction in energy consumption in constrained IoT devices.

In particular, we achieve scalability, decentralization and energy efficiency by adapting the IOTA Tangle and their consensus mechanism. We have presented the proposed approach in view of the architecture, consensus mechanism, and the computation offloading technique employed. 


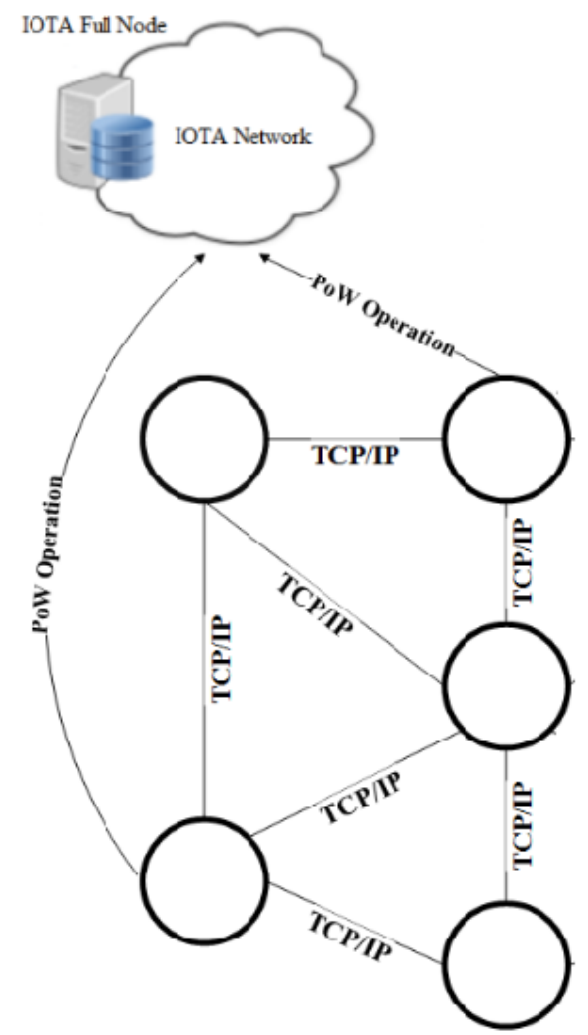

Fig. 2. Computation offloading in SDIT approach

\section{Experimental Results, Evaluation and Analysis}

In this section, we provide a description of the implementation of our experimental results, followed by an evaluation of the proposed solution in regard to the scalability, energy efficiency, and decentralization. Then, we conduct an analysis and discuss the results obtained, to highlight the useful characteristics of IOTA Tangle for IoT.

\subsection{Environment Setup}

We have deployed the latest release of IOTA Reference Implementation (IRI 1.8.1), which is the official Java build embodying the IOTA network specifications, ${ }^{1}$, on DigitalOcean cloud platform ${ }^{2}$, and another IOTA Reference Imple-

${ }^{1}$ https://github.com/iotaledger/iri/releases/tag/v1.8.1-RELEASE

${ }^{2}$ https://www.digitalocean.com 


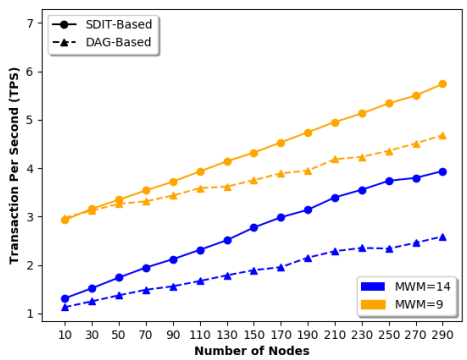

Fig. 3. Scalability in Tangle with 290 Nodes

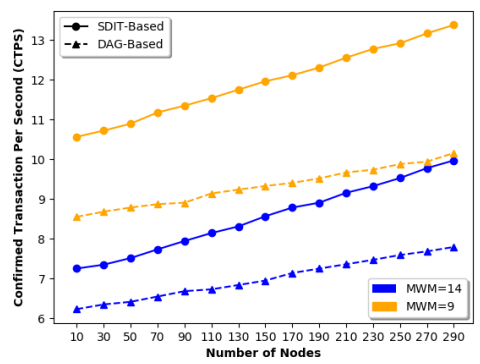

Fig. 4. Scalability in Tangle with 290 Nodes

mentation (IRI 1.8.1) on a local server dedicated for performing the operation of the Proof of Work (PoW).

The functionality related to IOTA addresses, transactions, broadcasting, routing, and multi-signatures has been implemented using iota.lib.py [33], the official Python library of IOTA Distributed ledger. In total, a large number of nodes with the specifications of medium size virtual machines (4GB RAM, 2 VCPU and 60.0GB Disk) are used to create the network. We have used medium size nodes and nodes with rich resources because this is more representative of real-world IoT scenarios. Nodes with rich resources enhance the performance by reducing the time it takes to perform the PoW.

In order to measure transaction speed and scalability, we configured each sending node to initiate a fixed number of transactions $=5$. We also used a set of different MWM $(9,11,13,14)$. These transactions are broadcasted among all nodes through TCP/IP.

We have tested the Transaction Per Second (TPS) and Confirmed Transaction Per Second (CTPS) under different numbers of nodes (50, 100, 150, up to $290^{3}$ ) with different MWM configurations as presented above, as shown in Figure 3 , and 4 respectively. TPS is defined as the number of transactions published to the network per second and CTPS is defined as the number of transactions that move from pending to confirmed per second.

\subsection{Results and Analysis}

In this part, we present the performance of the scalability and energy efficiency, which was evaluated over several runs to obtain accurate results. We compared it against one of the recent approaches in the literature, namely, DAG-based smart communities [29]. Their publications gave their full specifications, making it possible for researchers to implement and reproduce the published results. Finally, they achieved promising results for smart homes and are planning to extend their work with further comparisons.

\footnotetext{
${ }^{3}$ Due to resource constraints, we could only run up to 290 nodes.
} 


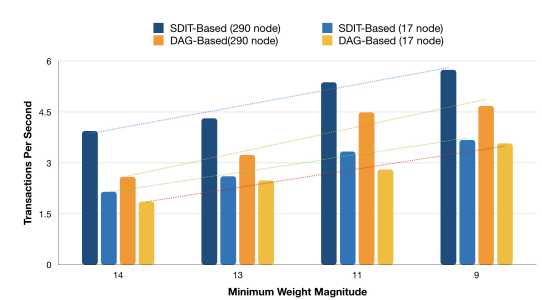

Fig. 5. Performance of TPS under different MWM

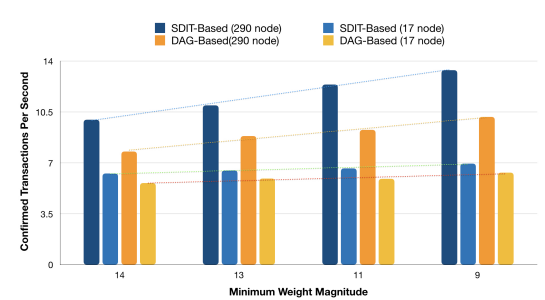

Fig. 6. Performance of CTPS under different MWM

Scalability: The results can be seen from Fig. 3 to Fig. 6. As shown in Fig. 3 and Fig. 4, when the MWM is set to 14, the TPS/CTPS results with a different number of nodes, it is clear that as the number of nodes increases, the TPS/CTPS transaction speed approximately increases linearly. Therefore, the transaction speed has good linear scalability when the number of nodes increases. For example, when 50 nodes are sending transactions, the STDI-Based approach TPS reaches $1.376 \mathrm{tx} / \mathrm{s}$ and CTPS $6.418 \mathrm{tx} / \mathrm{s}$ respectively, whereas with a DAG-Based approach the TPS reaches $1.743 \mathrm{tx} / \mathrm{s}$ and CTPS reaches 7.519 $\mathrm{tx} / \mathrm{s}$ respectively. This demonstrates that our proposed STDI-Based approach outperforms the DAG-Based approach and performs well when the number of nodes increases.

Throughput: As shown in Fig. 3 and Fig. 4, it is clear that our proposed STDI-Based approach outperforms the DAG-Based approach in terms of efficiency in processing transactions. For example, in the situation in which 10 nodes are sending, the average TPS reaches $1.132 \mathrm{tx} / \mathrm{s}$ and CTPS $6.234 \mathrm{tx} / \mathrm{s}$, respectively in STDI-Based approach. Whereas in the DAG-Based approach the TPS reaches $1.314 \mathrm{tx} / \mathrm{s}$ and CTPS reaches $7.256 \mathrm{tx} / \mathrm{s}$ respectively. This is due to the computation offloading mechanism used in STDI-Based approach.

Energy-Efficiency: The nodes involved in performing PoW have an impact upon total energy consumption. Therefore, computation offloading not only conserves energy but also improves the time to process transactions. A DAG-Based approach consumes more energy since IoT devices are required to perform the PoW as compared to our proposed STDI-based approach. Consequently, the STDI-Based approach outperforms a DAG-Based approach in terms of energy consumption.

The results in Fig. 5 and Fig. 6 are conducted to test the effect of MWM on the TPS and CTPS. In these experiments, we set the MWM to $9,11,13,14$ to measure the effect on the TPS/CTPS. In Fig. 5, it is clear that the TPS is affected by the use of different MWM, as when it is set to 13, it almost reaches $5.321 \mathrm{tx} / \mathrm{s}$ and when it is set to 14 , it almost reaches $6.591 \mathrm{tx} / \mathrm{s}$. From Fig. 6, the changes in MWM have almost no influence on the CTPS.

Decentralization: Our proposed SDIT-Based approach outperforms the DAG-Based approach in terms of decentralization. This is due to the use of the consensus mechanism in an SDTI-Based approach. 


\section{Conclusions and Future Work}

The work proposed in this paper is an important step towards the integration of IOTA Tangle DLT with the IoT. The results indicate that an IOTA Tangle can scale to a large number of IoT devices, thus addressing the scalability challenges in the IoT domain. IOTA Tangle can achieve considerable energy savings since IoT devices do not engage in performing the PoW. Compared to existing work, SDIT enables high-scalability and energy efficiency possibilities for building large-scale IoT applications.

There are a number of limitations in the paper so far that need to be addressed in the future, for example, the cost incurred by maintaining and deploying a dedicated servers for performing the PoW. There are several interesting directions for future work that we intend to follow.

First, we plan to investigate the usefulness of a Mobile Agent in assisting the Tangle in supporting DI i.e., delegating low-level intelligence to various network and application functions. For instance, IoT devices running the IOTA light node are not engineered to allow collaboration among these nodes in the network. This, in particular, would be useful for IoT applications, i.e., target tracking, where data needs to be shared among nodes to provide accurate location information.

Second, a Tangle can be used to solve the problem of offline capability. This task is not simply a network entities configuration problem; the major issue is related to clustering the network. However, it can be achieved by creating offline Tangles where a certain number of nodes can effectively go offline and issue transactions among themselves. This means that an active internet connection is not needed at all times for the Tangle to function. Upon completion, it is possible to attach the transactions of the offline Tangle back to the online one.

Third, we intend to explore the average confirmation rate of the transactions, which provides an insight into transaction time latency. This metric will be affected by the value of $\alpha$, the constant that affects confirmation rates. Therefore, an exploration will configure and test $\alpha$ under different scenarios to improve confirmation rates.

Finally, we are planning to explore the benefits offered by IOTA to other areas, such as Wireless Sensor Networks (WSN). It will not necessarily be pertinent to the scalability and energy-efficiency issues, and in particular, the work will focus on customising IOTA Tangle to drive an efficient routing protocol for IoT, taking into consideration various factors, such as Quality of Service. In addition to that, we shall investigate the possibility of adapting it to suit Information Extraction (IE) techniques in WSNs [34], and therefore not limiting the benefits of IOTA to a specific problem or problem domain.

\section{References}

[1] Luigi Atzori, Antonio Iera, and Giacomo Morabito. The internet of things: A survey. Computer Networks, 54(15):2787-2805, 2010.

[2] Cisco. Internet of things at a glance. (1), December 2016. 
[3] Gartner. Gartner says the internet of things installed base will grow to 26 billion units by 2020. (1), December 2013.

[4] API Research. More than 30 billion devices will wirelessly connect to the internet of everything in 2020. (1), May 2013.

[5] Tariq A. A. Alsboui, Yongrui Qin, and Richard Hill. Enabling distributed intelligence in the internet of things using the iota tangle architecture. In IoTBDS, 2019.

[6] Parker Lynne. Distributed intelligence: Overview of the field and its application in multi-robot systems. In The AAAI Fall Symposium Series. AAAI Digital Library, 2007.

[7] Satoshi Nakamoto et al. Bitcoin: A peer-to-peer electronic cash system. 2008.

[8] Popov Serguei. The tangle. (1), October 2017.

[9] Nabil El Ioini and Claus Pahl. A review of distributed ledger technologies. In Hervé Panetto, Christophe Debruyne, Henderik A. Proper, Claudio Agostino Ardagna, Dumitru Roman, and Robert Meersman, editors, On the Move to Meaningful Internet Systems. OTM 2018 Conferences, pages 277-288, Cham, 2018. Springer International Publishing.

[10] Andreas M. Antonopoulos. Mastering Bitcoin: Unlocking Digital CryptoCurrencies. O'Reilly Media, Inc., 1st edition, 2014.

[11] B. Cao, Y. Li, L. Zhang, L. Zhang, S. Mumtaz, Z. Zhou, and M. Peng. When internet of things meets blockchain: Challenges in distributed consensus. IEEE Network, pages 1-7, 2019.

[12] M. S. Ali, M. Vecchio, M. Pincheira, K. Dolui, F. Antonelli, and M. H. Rehmani. Applications of blockchains in the internet of things: A comprehensive survey. IEEE Communications Surveys Tutorials, 21(2):1676-1717, Secondquarter 2019.

[13] I-SCOOP. Blockchain and the internet of things: the iot blockchain opportunity and challenge. (1), Feb 2018. (visited on 19-09-2019).

[14] A. Dorri, S. S. Kanhere, and R. Jurdak. Towards an optimized blockchain for iot. In 2017 IEEE/ACM Second International Conference on Internetof-Things Design and Implementation (IoTDI), pages 173-178, April 2017.

[15] K. Christidis and M. Devetsikiotis. Blockchains and smart contracts for the internet of things. IEEE Access, 4:2292-2303, 2016.

[16] K. Biswas and V. Muthukkumarasamy. Securing smart cities using blockchain technology. In 2016 IEEE 18th International Conference on High Performance Computing and Communications; IEEE 14th International Conference on Smart City; IEEE 2nd International Conference on Data Science and Systems (HPCC/SmartCity/DSS), pages 1392-1393, Dec 2016.

[17] C. Fan, H. Khazaei, Y. Chen, and P. Musilek. Towards a scalable dag-based distributed ledger for smart communities. In 2019 IEEE 5th World Forum on Internet of Things (WF-IoT), pages 177-182, April 2019.

[18] N. K. Giang, M. Blackstock, R. Lea, and V. C. M. Leung. Developing iot applications in the fog: A distributed dataflow approach. In 2015 5th 
International Conference on the Internet of Things (IOT), pages 155-162, Oct 2015.

[19] Luis Alberto B. Pacheco, Eduardo Adílio Pelinson Alchieri, and Priscila América Solís Mendez Barreto. Device-based security to improve user privacy in the internet of things. In Sensors, 2018.

[20] Quang Duy La, Mao V. Ngo, Thinh Quang Dinh, Tony Q.S. Quek, and Hyundong Shin. Enabling intelligence in fog computing to achieve energy and latency reduction. Digital Communications and Networks, 5(1):3 9, 2019. Artificial Intelligence for Future Wireless Communications and Networking.

[21] Minh-Quang Tran, Duy Tai Nguyen, Van An Le, Duc Hai Nguyen, and Tran Vu Pham. Task placement on fog computing made efficient for iot application provision. Wireless Communications and Mobile Computing, 2019, 2019.

[22] C. Sarkar, A. U. Nambi S. N., R. V. Prasad, A. Rahim, R. Neisse, and G. Baldini. Diat: A scalable distributed architecture for iot. IEEE Internet of Things Journal, 2(3):230-239, June 2015.

[23] Bo Tang, Zhen Chen, Gerald Hefferman, Tao Wei, Haibo He, and Qing Yang. A hierarchical distributed fog computing architecture for big data analysis in smart cities. In Proceedings of the ASE BigData EZ\#38; SocialInformatics 2015, ASE BD\&\#38;SI '15, pages 28:1-28:6, New York, NY, USA, 2015. ACM.

[24] Higinio Mora, Maria Teresa Pont, David Gil, and Magnus Johnsson. Collaborative working architecture for iot-based applications. Sensors, 18:1676, 052018.

[25] Hussain Al-Aqrabi and Richard Hill. Dynamic multiparty authentication of data analytics services within cloud environments. In Proceedings - 20th International Conference on High Performance Computing and Communications, 16th International Conference on Smart City and 4th International Conference on Data Science and Systems, HPCC/SmartCity/DSS 2018, pages 742-749, United States, 1 2019. IEEE Computer Society.

[26] Ammar Muthanna, Abdelhamied A Ateya, Abdukodir Khakimov, Irina Gudkova, Abdelrahman Abuarqoub, Konstantin Samouylov, and Andrey Koucheryavy. Secure iot network structure based on distributed fog computing, with sdn/blockchain. 2019.

[27] Hussain Al-Aqrabi, Anju Johnson, and Richard Hill. Dynamic multiparty authentication using cryptographic hardware for the internet of things. In IEEE Smartworld Congress 2019, United States, 5 2019. IEEE Computer Society.

[28] Hussain Al-Aqrabi, Anju Pulikkakudi Johnson, Richard Hill, Philip Lane, and $\mathrm{Lu}$ Liu. A multi-layer security model for $5 \mathrm{~g}$-enabled industrial internet of things. In 7th International Conference on Smart City and Informatization (iSCI 2019), Guangzhou, China, November 12-15, 2019, Lecture Notes in Computer Science, Switzerland, 8 2019. Springer International Publishing AG. 
[29] C. Fan, H. Khazaei, Y. Chen, and P. Musilek. Towards a scalable dag-based distributed ledger for smart communities. In 2019 IEEE 5th World Forum on Internet of Things (WF-IoT), pages 177-182, April 2019.

[30] Kai Peng, Victor Leung, Xiaolong Xu, Lixin Zheng, Jiabin Wang, and Qingjia Huang. A survey on mobile edge computing: Focusing on service adoption and provision. Wireless Communications and Mobile Computing, 2018, 102018.

[31] IOTA Foundation. Minimum weight magnitude. (1), Nov 2017. (visited on 6-01-2019).

[32] Atis Elsts, Efstathios Mitskas, and George Oikonomou. Distributed ledger technology and the internet of things: A feasibility study. pages 7-12, 11 2018.

[33] IOTA Foundation. Pyota: The iota python api library. (1), Feb 2018. (visited on 8-08-2019).

[34] T Alsbouí, Mohammad Hammoudeh, Zuhair Bandar, and Andy Nisbet. An overview and classification of approaches to information extraction in wireless sensor networks. 2011. 